\title{
Ações de extensão universitária: educação nutricional para idosas
}

\author{
University extension actions: nutritional education for elderly
}

Franciele Coutinho França ${ }^{1}$ Leide da Conceição Sanches ${ }^{2}$ Maria Cecilia Da Lozzo Garbelini

1 Mestranda. Faculdades Pequeno Príncipe (FPP), Brasil. E-mail: framnutri2011@hotmail.com

2 Professora. Doutora. Faculdades Pequeno Príncipe (FPP), Brasil. E-mail: leide.sanches@fpp.edu.br

3 Professora. Doutora. Faculdades Pequeno Príncipe (FPP), Brasil. E-mail: maria.garbelini@fpp.edu.br

Recebido em: 18/11/2019 | Aprovado em: 20/02/2020

DOI: $10.12957 /$ interag.2019.53545

\section{Resumo}

A alimentação incorpora aspectos da vida humana, quais sejam emocionais, culturais e sociais, associados a momentos de prazer. O presente estudo se insere no âmbito da extensão universitária visando à educação nutricional para idosas. O objetivo do estudo foi promover atividade sobre educação nutricional para idosas em comunidade rural, por meio de troca de receitas trazidas pelas mesmas, para maior sensibilização e troca de experiências. Trata-se de uma pesquisa ação desenvolvida com um grupo de 130 idosas que se reúnem semanalmente no salão paroquial de uma cidade localizada na região metropolitana de Curitiba - PR. Para subsidiar as atividades educacionais, os extensionistas reuniram-se nos meses de agosto e setembro de 2018 para a elaboração de quatro atividades: pirâmide alimentar, orientação sobre o consumo de açúcar, gordura e sa encontrado em alimentos industrializados; modelo do prato saudável e paródia sobre alimentação saudável. O encontro com a comunidade ocorreu em outubro de 2018, por meio de uma prática reflexiva, com a de troca de saberes entre os acadêmicos extensionistas e as idosas. A atividade nutricional despertou vários questionamentos, não somente sobre a alimentação, mas também sobre patologias inerentes à idade tais como hipertensão e diabetes, bem como a rotina das participantes. A extensão possibilita a percepção da realidade e, por meio dela, a construção de novos e diferentes saberes a partir da fusão do conhecimento popular com o científico, além de favorecer a promoção da saúde a partir de um prisma holístico, com melhora na qualidade de vida, onde as diversas visões de mundo são respeitadas.

Palavras-chave: Educação em Saúde, Idosos, Hábitos Alimentares, Extensão Universitária.

\begin{abstract}
Eating incorporates various aspects of human life, as emotional, cultural and social, associated with moments of pleasure. The present study is part of the university extension aiming at nutritional education for the elderly. The aim of the study was to promote activity on nutrition education for elderly women in rural community, through the exchange of recipes brought by them, for greater awareness and exchange of experiences. This is an action research developed with a group of 130 elderly women who meet weekly in the parish hall of a city located in the metropolitan region of Curitiba - PR. To subsidize the educational activities, extensionists met in August and September 2018 to elaborate four activities: food pyramid, guidance on the consumption of sugar, fat and salt found in processed foods; Healthy dish template and spoof about healthy eating. The meeting with the community took place in October 2018, through a reflexive practice, with the exchange of knowledge between the extensionists and the elderly academics. The nutritional activity aroused many questions, not only about diet, but also about age-related pathologies such as hypertension and diabetes, as well as the participants' routine. The extension allows the perception of reality and, through it, the construction of new and different knowledge from the fusion of popular and scientific knowledge, and favoring health promotion from a holistic prism, with improved quality of life, where different worldviews are respected.
\end{abstract}

Keywords: Health Education; Elderly, Eating Habits, University Extension. 


\section{Introdução}

O artigo sobre ações de extensão universitária, cuja temática envolve educação nutricional para idosas, insere-se no projeto de extensão 'Educar par Prevenir', cujas atividades iniciaram-se em 2009 em uma Instituição de Ensino Superior (IES) de Curitiba e, desde então, são realizadas ininterruptamente em várias comunidades. Um dos campos de atuação do projeto é com um grupo de 130 senhoras idosas, em uma comunidade rural do Estado do Paraná, próximo a Curitiba. Neste contexto, o presente artigo foi construído mediante as ponderações acerca da importância nutricional, a partir da convivência dos docentes, mestrandos e acadêmicos extensionistas com a comunidade de idosas.

Segundo Moita e Andrade ${ }^{1}$, as atividades de extensão, pela indissociabilidade do ensinopesquisa-extensão, têm caráter orientador na qualidade de produção universitária e possibilitam que as IES desempenhem sua autonomia didático-científica. A tridimensionalidade torna-se necessária, para que se impedir os reducionismos na prática universitária.

Sabe-se que as atividades extensionistas complementam a formação profissional dos acadêmicos, pois possibilitam a aprendizagem em situação real e trazem mais significância à experiência. Os projetos de extensão são de suma importância à medida que possibilitam a percepção e o reconhecimento que a teoria deve ser desenvolvida e utilizada de acordo com a necessidade dos diferentes contextos. Por isso, a extensão universitária reconhecida como um dos três pilares da Educação Superior Brasileira representa um importante espaço de formação e de aproximação entre a universidade e a comunidade na qual ela está inserida. ${ }^{2,3}$

Ao integrar o processo formativo acadêmico com as comunidades, o que permite que haja trocas e relações com a realidade social, a extensão é espaço de avaliação crítica, para reconsiderar ações acadêmicas frente às demandas sociais e à formação de profissionais protagonistas de transformações sociais. ${ }^{4}$ 
Com base na importância da educação em saúde, a extensão tem se destacado como um terreno fértil para o desenvolvimento de ações junto às comunidades, que promovam práticas em saúde. Os extensionistas, ao participarem ativamente do processo pedagógico e, ao compreenderem suas responsabilidades, contribuem tomando decisões quanto à escolha dos temas, elaboração, execução e avaliação das atividades propostas. ${ }^{5}$

O foco da atividade extensionista desenvolvida neste estudo foi a promoção de práticas alimentares saudáveis com ações de educação alimentar e nutricional para as idosas, tendo em vista que o perfil demográfico da população brasileira vem se transformando desde o ano de 1970, com aumento relevante de pessoas com idade igual ou superior a 60 anos, fato esse que impacta na saúde e nos aspectos socioeconômicos do idoso. A cada ano são incorporados aproximadamente 650 mil idosos junto à população brasileira, sendo estimado para 2020 mais de 32 milhões de idosos. ${ }^{6,7}$

Segundo a Organização Mundial de Saúde (OMS) em países desenvolvidos considera-se como idoso o indivíduo que esteja com 65 anos ou mais de idade e para os países subdesenvolvidos o indivíduo a partir da faixa etária dos 60 anos ou mais de idade. ${ }^{8}$ Para os autores, o envelhecimento populacional nos países desenvolvidos teve inicio no final da década de 40 e início dos anos 50 em consequência da queda de mortalidade, urbanização, avanço da medicina, melhoria nutricional e aumento das condições de higiene pessoal e ambiental tanto em residências como no trabalho. Nos países menos desenvolvidos, o acréscimo da expectativa de vida tem sido evidenciado pelos avanços tecnológicos relacionados à área de saúde nas últimas seis décadas, como as vacinas, uso de antibióticos, quimioterápicos que tornaram possível a prevenção ou cura de muitas doenças.

A evolução das taxas de mortalidade, natalidade e fecundidade a partir de 1950 denota o processo de transição demográfica no Brasil passando de uma população majoritariamente jovem, em um passado recente, para um aumento de pessoas com 60 anos ou mais de idade. ${ }^{9}$ Ainda, a diminuição das taxas de mortalidade e natalidade faz 
com que o Brasil deixe de apresentar uma estrutura etária jovem, avançando para uma estrutura adulta e se direcionando para uma estrutura etária envelhecida. ${ }^{10}$

Conforme Mallmann e coautores ${ }^{11}$, pelas estatísticas mundiais o Brasil ganhou em 2013 a 80ª posição no ranking mundial da OMS, com a perspectiva média de vida entre 74 anos. Na primeira posição está o Japão com a perspectiva média de 83 anos.

Diante dos dados apresentados, reitera-se que a expansão da longevidade pode acarretar índices elevados de doenças crônicas não transmissíveis (DCNTs), perda da independência, depressão e má nutrição. Ainda, as condições peculiares do envelhecimento com enfermidades presentes, redução do metabolismo basal, alterações sensoriais, desordens do trato gastrointestinal e mastigação refletem diretamente na alimentação inadequada onde muitas das vezes o excesso ou déficit de nutrientes, favorece a desnutrição, o sobrepeso ou a obesidade. ${ }^{12}$ Por isso, o envelhecer com práticas saudáveis é importante para garantir a manutenção de uma melhor qualidade de vida. ${ }^{13}$

Em face às mudanças consequentes da idade e à perda progressiva de massa muscular, denominada como sarcopenia, ressalta-se a limitação da capacidade funcional e também a força mandibular no momento da ingestão de alimentos. Patologias como a osteopenia, relacionada à redução da estatura, a desidratação, ocasionada pela diminuição da água corporal, e a sarcopenia repercutem no estado nutricional da população geriátrica tornando-a mais vulnerável. Verifica-se que diversos são os fatores que podem interferir no estado nutricional da população idosa, o que pode acarretar alteração do olfato e das papilas gustativas, aumento do aporte proteico, deficiências na absorção da vitamina B6, B12, ferro, cálcio, ácido fólico e zinco, biodisponibilidade comprometida de vitamina D, xerostomia (conhecido como secura de boca) e intolerância à glicose. ${ }^{14}$

Por consequência das diversas alterações fisiológicas e anatômicas relacionadas à longevidade desta população a educação nutricional, em vários âmbitos, terá uma maior adesão e trará uma qualidade de vida mais digna, pois o decréscimo da ingestão e 
qualidade alimentar, ao passar dos dias, reflete posteriormente numa condição nutricional precária. ${ }^{15}$

No Brasil, as primeiras interferências do governo quanto à alimentação, determinadas pelas preocupações da ciência de nutrição, aconteceram por volta de 1940. Por influências de ordem política e ideológica, a educação alimentar nesse período estava centrada no binômio "alimentação e educação". Desta forma, os programas buscaram integrar a distribuição de alimentos a ações de caráter educativo, com o intuito de expandir os conhecimentos a respeito da alimentação racional e econômica da população. Entre 1940 a 1960, a educação alimentar e nutricional tornou-se alicerce das políticas de alimentação, relacionando-a junto a campanhas de introdução aos novos alimentos e métodos educativos. ${ }^{16,17}$

Já em 1970 o binômio "alimentação-educação" prevalente começou a ceder espaço para o binômio "alimentação-renda", vista como um dos obstáculos para adquirir uma alimentação saudável. Desse modo, por volta de 1980, consolida-se a educação nutricional crítica, com foco em contemplar não só as práticas alimentares, mas também esclarecer as pessoas sobre seus direitos. Cabe também reforçar que houve a discussão entre fome e desnutrição, em matrizes curriculares para a formação de nutricionistas. Como resultado, passa-se a debater a fome e não apenas a desnutrição, e desse modo, a educação alimentar passa a considerar não somente as práticas alimentares, mas também os direitos dos cidadãos. Nos anos de 1990 as discussões sobre segurança alimentar integraram o cenário internacional e nacional, concebendo a alimentação como um direito humano..$^{17,18,19}$

Assim, a instituição da Política Nacional de Alimentação e Nutrição (PNAN) pode ser avaliada como uma das expressões que oficializam a procura de uma nova direção das políticas de alimentação e nutrição no final da década de 1990. O propósito da PNAN é a garantia da qualidade dos alimentos colocados para consumo no País, a promoção das práticas alimentares saudáveis e a prevenção dos distúrbios nutricionais, bem como o estímulo às ações intersetoriais que propiciam o acesso universal aos alimentos. ${ }^{20}$ 
Após a vigência da Política Nacional de Alimentação e Nutrição em 1999 e renovação em 2011, a educação nutricional e alimentar passou a ser um método de trocas de informações entre os profissionais nutricionistas e a população assistida, levando em consideração a heterogeneidade, desenvolvendo práticas de prevenção, promoção e monitoramento nutricional. ${ }^{21,22}$

A transição epidemiológica nutricional destaca a redução ou desaparecimento de carências graves de vitaminas e minerais, associadas a doenças infecciosas, endemias e aparecimento epidêmico de carência nutricional, refletindo num alto nível de morbimortalidade no passado. Conforme as alterações da base demográfica da pirâmide populacional a longevidade está crescendo, porém em paralelo ocorreram mudanças dos hábitos alimentares e estilo de vida, assim, configura-se um novo cenário epidemiológico, no qual se evidencia a redução da mortalidade. No entanto, cresce o número de comorbidades associadas às doenças crônicas não transmissiveis (DCNT). ${ }^{23}$

Com respeito às necessidades nutricionais específicas para idosos, a literatura atual aponta para a educação em saúde como subsídio e ferramenta positiva, na construção de conhecimento para o autocuidado, e deve ser usada pelos futuros profissionais que trabalham com esta clientela. A alimentação adequada nessa fase da vida é de suma importância para a saúde física e mental, pois permite o melhoramento da capacidade funcional de cada pessoa. ${ }^{24}$

Reitera-se que, fazer educação nutricional é respeitar a autonomia do indivíduo, valorizar suas crenças, aspectos sociais e etnias, pois o mesmo deve ser o protagonista das ações educativas, contrapondo a sua realidade e promovendo mudanças nos hábitos alimentares no âmbito familiar. ${ }^{25}$ É nessa perspectiva que se situa o presente trabalho, cujo objetivo foi promover atividade sobre educação nutricional para idosas em comunidade rural, por meio de troca de receitas trazidas pelas mesmas, para maior sensibilização e troca de experiências. 


\section{Metodo}

Trata-se de uma pesquisa-ação com abordagem qualitativa, desenvolvida para um grupo de 130 idosas, que se reúne semanalmente no salão paroquial de uma cidade localizada na região metropolitana de Curitiba. Nos encontros semanais, o grupo realiza atividades recreativas organizadas pelo Sistema Único de Assistência Social (SUAS) em parceria com a Secretaria de Saúde do município, responsável pela divulgação das atividades e pelo transporte das participantes. Os encontros são acompanhados por profissionais ligados ao SUAS, psicóloga e assistente social, com o intuito de pensar a política de Assistência Social e produzir contribuições para a sua efetivação. O comparecimento das idosas gira em torno de $80 \%$, quantificado por uma lista de presença apresentada a cada encontro.

A pesquisa-ação é um tipo de pesquisa social idealizada e dirigida em estreita associação com uma ação onde os pesquisadores e os participantes da pesquisa estão envolvidos de modo cooperativo e participativo em uma relação dialética. ${ }^{26}$ Já a abordagem qualitativa, baseia-se no princípio de que o conhecimento dos processos sociais só é possível com a descrição da experiência humana, tal como vivida e definida por seus próprios atores. ${ }^{23}$ Para a condução da pesquisa-ação foram executadas três fases: planejamento, intervenção e avaliação com base nas fases descritas por Thiollent. ${ }^{27}$

Ao iniciar a primeira fase, a escolha do tema, foi perguntado previamente para a comunidade participante qual seria o assunto de interesse para o encontro que aconteceria em outubro de 2018. Ressalta-se que o Projeto Educar para Prevenir faz um trabalho contínuo, semestral, com o grupo de idosas. Foi escolhido o tema Alimentação Saudável e, para subsidiar as atividades educacionais, os extensionistas, mestrandos e os pesquisadores reuniram-se nos meses de agosto e setembro de 2018 para a elaboração do planejamento de cinco atividades: Pirâmide alimentar; Orientação sobre o consumo de açúcar, gordura e sal encontrados em alimentos industrializados; Modelo do prato saudável; Paródia sobre alimentação saudável e Elaboração de um caderno de receitas. 
Estas reuniões foram supervisionadas por uma nutricionista e aconteceram a cada semana, totalizando dez encontros.

Para cumprir as etapas de intervenção e avaliação o encontro com a comunidade ocorreu em outubro de 2018, por meio de uma prática reflexiva, com o objetivo de troca de saberes entre todos os envolvidos e com foco nas necessidades sociais vinculadas ao meio natural de vida do grupo participante. ${ }^{28}$ A pesquisa-ação, no uso da estratégia de intervenção, foi iniciada por uma roda de conversa que evoluiu para um contexto dinâmico, tendo a nutricionista como facilitadora do processo. A técnica de natureza dialética sobre o tema foi usada para o esclarecimento das dúvidas e dificuldades das participantes no que se refere ao acesso ao alimento, à qualidade do alimento consumido nas refeições, e aos efeitos de uma nutrição inadequada. Na sequência as participantes foram divididas em equipes, para a execução da dinâmica que consistia na construção do prato saudável, para uma refeição diária, com modelos de alimentos construídos com EVA.

$\mathrm{Na}$ fase de avaliação foram discutidos os benefícios e os resultados das ações educativas, onde uma representante de cada equipe explicou a importância de cada alimento que estava presente no prato elaborado com moldes de EVA. No decorrer das ações educativas, houve a preocupação de usar uma linguagem acessível, bem como relacionar os conteúdos à rotina diária das participantes.

A pesquisa obedeceu aos preceitos éticos conforme determina a Resolução 466/12 do Conselho Nacional de Saúde (CNS), no que diz respeito à pesquisa com seres humanos. ${ }^{29} \mathrm{O}$ projeto foi encaminhado ao Comitê de Ética da IES para assegurar que a pesquisa fosse eticamente aceitável e que os direitos das participantes estivessem salvaguardados. A pesquisa teve início logo após a autorização do Comitê de Ética em Pesquisa (CEP), sob Parecer Consubstanciado no 2.827 .939 e, para amparar os aspectos éticos, foi fornecido as participantes o Termo de Consentimento Livre e Esclarecido (TCLE) para que assinassem sua conformidade na participação da pesquisa. 


\section{Resultados e Discussão}

A Resolução no 7, de 18 de dezembro de 2018, em seu Art. 5 e parágrafo primeiro organiza o exercício das Diretrizes da Extensão na Educação Superior frente à interação dialógica dos acadêmicos com a sociedade por meio da troca de conhecimentos, da participação e do contato com as questões complexas e atuais presentes no contexto social. $^{30}$ Atendendo a Resolução citada, o encontro dos extensionistas com as idosas, objetivando trabalhar a saúde nutricional ligada à realidade de cada uma, despertou vários questionamentos decorrentes não somente da alimentação, mas também sobre as patologias inerentes à idade e também à alimentação, como hipertensão e diabetes. Teve como meta a promoção da saúde por meio da sensibilização e troca de experiências com os envolvidos, proporcionando momentos de aprendizagem sobre a alimentação saudável, descontração e prazer.

Escolhas alimentares exercem papel relevante ao longo da vida e, se pautadas em opções inadequadas, podem desencadear doenças. Com o crescimento da população idosa há um aumento das DCNTs próprias das faixas etárias mais avançadas que, em relação aos idosos residentes na zona rural, atinge cerca 75,5\% desta população. ${ }^{31}$ Várias barreiras podem contribuir para o aumento da vulnerabilidade dos idosos tais como as grandes distâncias a serem percorridas em busca dos serviços de saúde, dificuldade de transporte, baixa renda e nível reduzido de escolaridade. ${ }^{32}$ Portanto, é fundamental planejar e desenvolver ações de saúde, voltadas a uma alimentação saudável, que possam colaborar com a melhoria da qualidade de vida desta parcela populacional.

As atividades foram apresentadas com a finalidade de elucidar, de forma dinâmica, os hábitos alimentares mais saudáveis no dia-a-dia. Vale ressaltar que a baixa escolaridade de algumas participantes poderia ser um fator limitante para a compreensão e assimilação da temática apresentada. Para vencer este obstáculo a comunicação oral foi objetiva, pausada e com uma linguagem de fácil entendimento. Foram cumpridas as seguintes etapas: 


\section{Atividade 1 - Apresentação da Pirâmide Alimentar}

Adaptada por Philippi e colaboradores ${ }^{33}$, a pirâmide alimentar é um guia alimentar importante e pode ser usada como ferramenta nas atividades de educação nutricional para demonstrar as porções recomendadas de cada grupo alimentar. Demonstra ainda como optar diariamente pelos alimentos mais saudáveis, relacionando-os com a prevenção de doenças ao longo dos anos. ${ }^{33,34}$

Realizou-se uma apresentação e explicação, por meio de uma réplica da pirâmide alimentar, com a definição e divisão de cada grupo alimentar utilizado no dia a dia. Falouse do grupo de cereais, pães, tubérculos, raízes e massas; hortaliças e frutas; leites, derivados e leguminosas; carnes e ovos; óleos e gorduras; açúcares e doces. O objetivo da atividade foi mostrar a quantidade recomendada de cada grupo alimentar, além da importância da escolha certa dos alimentos para a saúde. Ressalta-se que as participantes enviaram previamente suas receitas preferidas para a equipe do projeto, as quais incluíram todos os grupos alimentares trabalhados na atividade de extensão.

Os aspectos relacionados com a educação nutricional são amplos frente à tríade do comer, alimentar e nutrir, pois enovela fatores socioculturais, biológicos, ambientais, entre outros. Para tanto, a Educação Alimentar e Nutricional (EAN) de 2012 propõe princípios a serem seguidos ao planejar e desenvolver ações que colocam como protagonistas as pessoas inseridas, seja lá qual for o cenário. ${ }^{35,36}$

De acordo com Fazzio ${ }^{37}$ e Lima $^{38}$ a alimentação saudável deve ser embasada nas quatro leis elaboradas pelo médico argentino Pedro Escudero, em 1937. Dentre as leis estão citam-se a quantidade e a qualidade dos alimentos, isto é, saber fazer as escolhas para suprir a demanda do organismo, lembrando que cada indivíduo possui necessidades diferenciadas e, por fim, têm-se as leis da harmonia e adequação que nada mais são do que equilibrar e adequar todos os nutrientes de acordo com a faixa etária, gênero ou em qualquer condição que o organismo se encontre. 
Portanto, antes de alcançar uma nutrição saudável, o ato de alimentar-se é necessário e depende totalmente, da autonomia do indivíduo em suas escolhas ao selecionar a qualidade e quantidade do alimento no seu dia a dia. Posteriormente, após a eleição do alimento, inicia-se o processo da nutrição no momento em que o mesmo é levado a boca e, em seguida passará pelo trato gastrointestinal para a digestão, tendo como parte final a absorção dos nutrientes. ${ }^{39}$

\section{Atividade 2 - Orientação sobre o consumo de açúcar, gordura e sal}

Sabe-se que o consumo excessivo de alimentos com alto teor de açúcar, gorduras e sal podem ser prejudiciais à saúde, além de serem responsáveis pela incidência de doenças cardiovasculares, diabetes, sobrepeso e obesidade. Pelos motivos expostos, salienta-se a importância de realizar atividades educacionais que abordam temáticas direcionadas às mudanças de hábitos alimentares.

Com base em alimentos industrializados, foi demonstrada a quantidade de açúcar, gordura e sal contida em "cada porção e no total da embalagem", conforme a informação nutricional disponível no rótulo de cada alimento. Para desenvolver este tópico utilizaram-se produtos industrializados consumidos pela população em geral, como refrigerante em lata, suco de pacote, macarrão instantâneo, biscoito recheado, biscoito salgado, salgadinho de pacote, pipoca doce de pacote e pipoca para micro-ondas. O objetivo foi apresentar de forma lúdica o prejuízo à saúde, ao longo dos anos, causado pelo consumo em excesso destes alimentos. Foram enfatizadas doenças como a hipertensão, diabetes e hipercolesterolemia.

A alimentação e nutrição são quesitos indispensáveis na vida do ser humano, sendo reconhecida pela Constituição Federal brasileira como um direito humano universal, porém, é necessário atentar-se para a qualidade e benefício que o alimento proporcionará ao indivíduo. ${ }^{40} \mathrm{~A}$ escolha do alimento saudável reflete melhor qualidade de vida, sendo uma das medidas preventivas para o aparecimento ao longo dos anos de doenças crônicas 
não transmissíveis (DCNT), como diabetes, hipertensão, dislipidemias, obesidade, doenças cardiovasculares, entre outras. ${ }^{41}$

De forma crescente e prevalente, as DCNT são também mundialmente responsáveis pela morbidade e mortalidade, como reflexo das escolhas e práticas diárias dos indivíduos. São geradoras, em longo prazo, do comprometimento de lesões e complicações no que se refere à saúde dos indivíduos o que pode favorecer a incapacidade ou mesmo óbito., ${ }^{42,43}$

\section{Atividade 3 - Modelo do Prato saudável}

O modelo proposto do prato saudável demonstrou a utilização dos alimentos recomendados para obter uma refeição colorida e equilibrada em nutrientes importantes. Deve ser composto por $50 \%$ de verduras e legumes, responsáveis pelo aporte de vitaminas e minerais; $25 \%$ composto por proteínas (carnes, peixes ou ovos) e, por fim, $25 \%$ composto por carboidratos (arroz ou massas integrais ou tubérculos).

Para esta atividade foi explicado que os nutrientes se dividem em dois grupos: macro e micronutrientes, os quais são responsáveis em promover uma nutrição balanceada ao organismo. No primeiro grupo estão os carboidratos, proteínas e lipídios enquanto as vitaminas e minerais pertencem ao segundo grupo, ou seja, os micronutrientes. $\mathrm{O}$ segundo grupo exerce papel importante mediante uma alimentação mista em quantidade, suprindo todas as necessidades do indivíduo ao longo do dia. ${ }^{44}$

Para a explicação de como elaborar um prato saudável foram utilizados jogos de quebra cabeça, confeccionados em MDF, e imagens de alimentos desenvolvidos em EVA tais como arroz branco, arroz integral, feijão, macarrão, batata frita, carne grelhada, ovos, verduras e legumes. Havia a opção de preparo de pratos saudáveis ou não. Após a explicação realizada pela equipe do projeto, em uma roda de conversa, as participantes foram divididas em seis equipes e orientadas para que montassem um prato com porções adequadas de cada grupo alimentar. A atividade teve como objetivo mostrar de forma lúdica como seria um prato saudável e equilibrado. 
Henn e colaboradores ${ }^{45}$ também realizaram atividade com idosos, com base no prato saudável, onde o grupo alvo demonstrou os hábitos alimentares no dia a dia e, ao mesmo tempo, as pesquisadoras puderam esclarecer dúvidas em relação às escolhas quanto ao modo de preparo dos alimentos, distribuição, quantidade e composição das refeições.

Os aspectos econômicos, sociais e culturais de cada indivíduo devem ser levados em consideração para alcançar uma nutrição saudável, que envolva o equilíbrio, a diversidade e a harmonia nos quesitos quantidade e qualidade alimentar. Em contrapartida, saber respeitar a autonomia nas escolhas dos alimentos e criar estratégias em educação nutricional e alimentar, facilitará a prática de hábitos alimentares mais saudáveis. ${ }^{46}$

Acredita-se que a informação/mudança alimentar foi compreendida pelos grupos como necessária, tanto a partir dos esclarecimentos iniciais feitos pelas pesquisadoras e extensionistas, como pela dinâmica realizada com as idosas. Em decorrência das atividades planejadas na pesquisa-ação, com o uso de instrumentos de natureza dialética, criou-se uma oportunidade de valorização na construção da experiência, apoiada na reflexão crítica e no feedback direto/imediato sobre a importância nutricional dos alimentos para uma vida saudável. Nesse sentido, orientar a necessidade nutricional de acordo com a faixa etária é muito importante visto que, com o aumento no ritmo de envelhecimento da população, torna-se essencial planejar e desenvolver ações de saúde que possam contribuir com a melhoria da qualidade de vida dos idosos brasileiros.

\section{Atividade 4 - Paródia Alimentação Saudável}

Finalizando a ação educativa os alunos extensionistas apresentaram uma paródia com o tema alimentação saudável, tornando o momento descontraído e interagindo com as idosas. Teve por objetivo incentivar a reflexão sobre o conteúdo trabalhado e a aquisição de hábitos mais saudáveis, o que pode contribuir para a redução do risco de desenvolvimento das DCNTs. 
Posteriormente foi elaborado um receituário ilustrado e informativo, em forma de um caderno de receitas, com base no material enviado previamente pelas senhoras, com o propósito de resgatar a cultura alimentar do grupo alvo. Nesse manual de receitas constaram os pratos que as idosas mais apreciavam. Nas receitas simples, saborosas e nutritivas as pesquisadoras enfatizaram os cuidados gerais com os alimentos com valorização do conhecimento e da cultura regional.

\section{Considerações Finais}

Privilegiou-se neste estudo a importância de práticas alimentares saudáveis onde a comunidade acadêmica, por meio da equipe que realizou a atividade de extensão, atuou como facilitadora na aproximação com a comunidade. As informações advindas da troca de experiência forneceram matéria- prima aplicável para a promoção e prevenção em saúde. As atividades sobre alimentação saudável despertaram vários questionamentos, não somente sobre a alimentação em si, mas sobre patologias inerentes à idade e aos hábitos alimentares, tais como hipertensão e diabetes, bem como sobre o dia a dia das senhoras.

O desenvolvimento de ações junto à comunidade possibilitou perceber melhor a realidade e, por meio dela, a construção de novos e diferentes saberes, a partir da fusão do conhecimento popular com o científico. As ações extensionistas promoveram a garantia do intercâmbio de conhecimentos e o envolvimento com questões sociais, promovendo a adesão quanto à possibilidade de mudanças alimentares.

Assim, frente aos problemas de saúde comuns entre os idosos a aplicação do método mostrou-se adequada com a necessidade de assimilação de novas informações e valores. Salienta-se a necessidade de apreender as experiências e o conhecimento acumulado pelo público- alvo do projeto, para conseguir respostas positivas que pudessem influenciar na mudança de hábito. A prática extensionista permitiu melhoria para a comunidade 
assistida e, ao mesmo tempo, a vivência na extensão contribuiu para a reflexão e o enriquecimento da formação profissional.

\section{Referências bibliográficas}

1. MOITA, Filomena Maria Gonçalves da Silva Cordeiro; ANDRADE, Fernando Cézar Bezerra de. Ensino-pesquisa-extensão: um exercício de indissociabilidade na pós-graduação. Universidade Federal da Paraíba, Departamento de Fundamentação da Educação. Revista Brasileira de Educação, v. 14 n. 41 maio/ago. 2009.

2. POSSER, Juliana; PEDROSO, Débora. Ensino, Pesquisa e Extensão: a Educação em Saúde como ferramenta para prevenção de parasitoses. Cataventos Revista de Extensão da Universidade de Cruz Alta, v. 8, n. 01, p. 74-89, 2016.

3. BARRAGÁN, Tereza Ontañón; RODRIGUES, Gilson Santos; SPOLAOR Gabriel da Costa e BORTOLETO, Marco Antonio Coelho. O PAPEL DA EXTENSÃO UNIVERSITÁRIA E SUA CONTRIBUIÇÃO PARA A FORMAÇÃO ACADÊMICA SOBRE AS ATIVIDADES CIRCENSES. Pensar a Prática, Goiânia, v. 19, n. 1, jan./mar. 2016.

4. SILVA, Antonio Fernando Lyra da; RIBEIRO, Carlos Dimas Martins; SILVA JÚNIOR, Aluísio Gomes. Pensando extensão universitária como campo de formação em saúde: uma experiência na Universidade Federal Fluminense. Interface (Botucatu), v.17, n.45, p.371-84, abr./jun. 2013.

5. RIOS, David Ramos da Silva; CAPUTO, Maria Constantina. Para Além da Formação Tradicional em Saúde: Experiência de Educação Popular em Saúde na Formação Médica. Revista Brasileira de Educação Médica, v. 43, n. 3, p. 184-195, 2019.

6. MIRANDA, Gabriella Morais Duarte; MENDES, Antonio da Cruz Gouveia; SILVA, Ana Lucia Andrade. O envelhecimento populacional brasileiro: desafios e 
consequências. Rev. Bras. Geriatr. Gerontol, Rio de Janeiro, v. 3, n. 19, p. 507 519, 2016.

7. SILVA, João Victor Farias da; SILVA, Edlla Cabral da; RODRIGUES, Ana Paula Rebelo Aquino; MIYAZAWA, Ana Paula. A relação entre o envelhecimento populacional e as doenças crônicas não transmissíveis: Sério desafio de saúde pública. Ciências Biológicas e da Saúde, Maceió, v. 2, n. 3, p. 91-100, 2015.

8. MENDES, Márcia Regina Silvério Santana Barbosa; GUSMÃO, Josiane Lima de; FARO, Ana Cristina Mancussi e; LEITE, Rita de Cássia Burgos de O. A situação social do idoso no Brasil: uma breve consideração. Acta Paul Enferm, v. 18. n. 4, p. $422-426,2005$.

9. WONG, Laura Rodrigues; CARVALHO, José Alberto M. Age-strucutural transition in Brazil: demographic bonuses and emerging challenges. In: Pool I, Wong LLR, Vilquin E, editores. Age-structural transitions: challenges for development. Paris: Committee for International Cooperation in National Research in Demography; 2006.

10. BOSCARDIN, Mariele; SPANEVELLO, Rosani Marisa. Dinâmica demográfica da população rural: um estudo de caso na microrregião e município de Frederico Westphalen/RS. Extensão Rural DEAER - CCR - UFSM Santa Maria, v. 25, n. 2, abr./jun. 2018.

11. MALLMANN, Danielli. Gavião; GALINDO NETO, Nelson Miguel; SOUSA, Josueida Carvalho Souza; VASCONCELOS, Eliane Maria Ribeiro. Educação em saúde como principal alternativa para promover a saúde do idoso. Ciência \& Saúde Coletiva, v. 20, n. 6, p. 1763-1772, 2015.

12. CAMPOS, Maria Teresa Fialho de Sousa; MONTEIRO, Josefina Bressan Resende; ORNELAS, Ana Paula Rodrigues de Castro. Fatores que afetam o consumo alimentar e a nutrição do idoso. Rev. Nutr., Campinas, v. 13, n. 3, p. 157-165, set./dez. 2000.

13. DAWALIBI, Nathaly Wehbe; ANACLETO, Geovana Mellisa Castrezana; 
WITTER, Carla; GOULART, Rita Maria Monteiro; AQUINO, Rita de Cássia de; Envelhecimento e qualidade de vida: análise da produção científica da SciELO. Estudos de Psicologia, Campinas, v. 30, n. 3, p. 393-403, 2013.

14. SANTOS, Ana Célia Oliveira dos. MACHADO, Myrtes Maria de Oliveira; LEITE, Elder Machado. Envelhecimento e alterações do estado nutricional. Geriatria \& Gerontologia, v. 4, n. 81, p. 168-175, 2010.

15. BUENO, Júlia Macedo; MARTINO, Hercia Stampini Duarte; FERNANDES, Maria Fernanda Scareli; COSTA, Luciana Silva; SILVA, Roberta Ribeiro. Avaliação nutricional e prevalência de doenças crônicas não transmissíveis em idosos pertencentes a um programa assistencial. Ciênc. saúde coletiva, v. 13, n. 4, p. 1237-1246 jul./ago. 2008.

16. CASTRO, C; PELIANO, A. M. Novos alimentos, velhos hábitos e o espaço para ações educativas. In: CASTRO; COIMBRA (Orgs.). O problema alimentar no Brasil. 1. ed. São Paulo: Unicamp, 1985. p. 195-213.

17. SANTOS, Ligia Amparo da Silva. Educação alimentar e nutricional no contexto da promoção de práticas alimentares saudaveis. Rev. Nutr., v. 18, n. 5, p. 681692, 2005.

18. CERVATO-MANCUSO, Ana Maria; VINCHA Kellem Regina Rosendo; SANTIAGO, Débora Aparecida. Educação Alimentar e Nutricional como prática de intervenção: reflexão e possibilidades de fortalecimento. Physis Revista de Saúde Coletiva, Rio de Janeiro, v. 26, n. 1, p. 225-249, 2016.

19. LIMA, Eronides da Silva; OLIVEIRA, Celina Szuchmacher; GOMES, Maria do Carmo Rebello. Educação nutricional: da ignorância alimentar à representação social na pós-graduação do Rio de Janeiro, 1980-1998. História Ciência Saúde, Manguinhos, Rio de Janeiro, v. 10, n. 2, p. 604-35, 2003.

20. BRASIL. Ministério da Saúde. Política Nacional de Alimentação e Nutrição. Brasília: Ministério da Saúde; 2000. 
21. BRASIL. Ministério da Saúde. Política Nacional de Alimentação e Nutrição. Brasília: Ministério da Saúde, n. 2, p. 1-48, 2003.

22. PACHECO, Pauline Muller; RAMOS, Mauren. Nutricionista em atenção básica: a visão do usuário. Demetra: alimentação, nutrição \& saúde, Rio Grande do Sul, v. 9, n. 2, p. 483-501, 2014.

23. BURLANDY, Luciene; COSTA, Rosana Salles. Segurança alimentar e nutricional: concepções e desenhos de investigação. In: KAC, G.; SICHIERI, R.; GIGANTE, D. P. Epidemiologia Nutricional. Rio de Janeiro: Ed. Fiocruz, 2007. p. 485-502.

24. 24. BERNARDI, Ana Paula. MACIEL, Manuela Avila. BARATTO, Indiomara. EDUCAÇÃO NUTRICIONAL E ALIMENTAÇÃO SAUDÁVEL PARA ALUNOS DA UNIVERSIDADE ABERTA A TERCEIRA IDADE (UNATI). Revista Brasileira de Obesidade, Nutrição e Emagrecimento, v. 11, p. 224-231, 2017.

25. TEIXEIRA, Pryscila Dryelle Sousa; REIS, Bruna Zavarize; VIEIRA, Diva Aliete dos Santos; COSTA, Dayanne da; COSTA, Jamille Oliveira et al. Intervenção nutricional educativa como ferramenta eficaz para mudança de hábitos alimentares e peso corporal entre praticantes de atividade física. Ciência \& Saúde Coletiva, v. 18, n. 2, p. 347-356, 2013.

26. THIOLLENT Michel. Metodologia da pesquisa-ação. 13. ed. São Paulo: Cortez; 2004.

27. POLITY Denise F; HUNGLER Bernadete P; BECK Cheryl Tatano. Fundamentos de pesquisa em enfermagem. 3. ed. Porto Alegre: Artes Médicas; 2004.

28. FRANCO, Maria Amélia Santoro. Pedagogia da Pesquisa-Ação. Educação e Pesquisa, São Paulo, v. 31, n. 3, p. 483-502, set./dez. 2005. 
29. BRASIL. Ministério da Saúde. Resolução 466/2012 do Conselho Nacional de Saúde, que institui. Diretrizes e Normas Regulamentadora das Pesquisas envolvendo seres humanos. Brasília: Ministério da Saúde, 2012.

30. BRASIL. Ministério da Educação. Resolução no 7, de 18 de dezembro de 2018 que Estabelece as Diretrizes para a Extensão na Educação Superior Brasileira e regimenta o disposto na Meta 12.7 da Lei no 13.005/2014, que aprova o Plano Nacional de Educação - PNE 2014-2024 e dá outras providências, p. 1-12, 2018.

31. VERAS, Renato; PARAHYBA, Maria lsabel. O anacronismo dos modelos assistenciais para os idosos na área da saúde: desafios para o setor privado. Cad Saúde Pública. V. 23, n. 10, p. 2479-89, 2007.

32. MORAIS, Eliane Pinheiro; RODRIGUES, Rosalina Aparecida Partezani; GERHARDT Tatiana Engel. Os idosos mais velhos no meio rural: realidade de vida e saúde de uma população do interior gaúcho. Texto Contexto Enferm. v. 17, n. 2, p. 374-83, abr-jun. 2008.

33. PHILIPP, Sonia Tucunduva; LATTERZA, Andrea Romero; CRUZ, RIBEIRO, Ana Teresa Rodrigues Luciana Cisotto. PIRÂMIDE ALIMENTAR ADAPTADA: GUIA PARA ESCOLHA DOS ALIMENTOS. Rev. Nutr. Campinas, v. 12, n. 1, p. 65-80, jan./abr. 1999.

34. MENDES-NETTO, Raquel Simões; TEIXEIRA, Pryscila Dryelle Sousa; REIS, Bruna Zavarize; VIEIRA, Diva Aliete Santos; COSTA, Dayanne da; COSTA, Jamille Oliveira. Ações Educativas para Promoção de Hábitos Alimentares Saudáveis : relato de uma experiência. Revista de Extensão Universitária da UFS, p. 191-199, 2013.

35. BRASIL. Ministério do Desenvolvimento Social. Princípios e Práticas para Educação Alimentar e Nutricional, Brasília: Ministério do Desenvolvimento Social, 2018.

36. SANTOS, Lígia Amparo. Avanços e desdobramentos do marco de referência da educação alimentar e nutricional para políticas públicas no âmbito da 
universidade e para os aspectos culturais da alimentação. Ver. Nutr., v. 26, p. 595-600, 2013.

37. FAZZIO, Débora Mesquita Guimarães; Envelhecimento e qualidade de vida uma abordagem nutricional e alimentar. Revisa, v. 1, n. 61, p. 76-78, 2012.

38. LIMA, Eronides da Silva. Quantidade, qualidade, harmonia e adequação: princípios-guia da sociedade sem fome em Josué de Castro. História, Ciências, Saúde, Manguinhos, Rio de Janeiro, v. 16, n. 1, p. 171-194, jan./mar. 2009.

39. BRASIL. Ministério da Educação; Alimentação e Nutrição no Brasil. Brasília: Ministério da Educação, 2009.

40. FERREIRA, Vanessa A.; MAGALHÃES, Rosana. Nutrição e promoção da saúde: perspectivas. Nutrição e Promoção da Saúde, Rio de Janeiro, v. 23, n.7, p. 1674 $1681,2007$.

41. SICHIERI, Rosely; COITINHO. Denise C; MONTEIRO, Josefina B; COUTINHO, Walmir F. Recomendações de Alimentação e Nutrição Saudável para a População Brasileira. Arq Bras Endocrinol Metab, Rio de Janeiro, v. 44, n. 3, p. 227-232, 2000.

42. THEME FILHA, Mariza Miranda; SOUZA JUNIOR, Paulo Roberto Borges de; DAMACENA, Giseli Nogueira; SZWARCWALDI, Celia Landmann. Prevalência de doenças crônicas não transmissíveis e associação com autoavaliação de saúde: Pesquisa Nacional de Saúde, 2013. Rev Bras Epidemiol. v. 18, n. 2. p. 83-96, dez. 2015.

43. MARIATH, Aline Brandão; GRILLO, Luciane Peter; SILVA, Raquel Oliveira da; SCHMITZ, Patrícia; CAMPOS, Isabel Cristina de; MEDINA, Janete Rosa Pretto; KRUGER, Rejane Magda. Obesidade e fatores de risco para o desenvolvimento de doenças crônicas não transmissíveis entre usuários de unidade de alimentação e nutrição. Cad. Saúde Pública, Rio de Janeiro, v.23, n. 4, p. 897-905, abr. 2007. 
44. Sociedade Brasileira de Diabetes (SBD). Manual de Nutrição Profissional da Saúde. São Paulo: Departamento de Nutrição e Metabologia da SBD; 2009.

45. HENN, Rosana; ALMEIDA, Elisabeth Barth; GUEDES, Mariana Reiser; SANTOS, Alexandra Christiane Cabral dos; OTT, Ana Paula Corteze et al. EDUCAÇÃO ALIMENTAR E NUTRICIONAL PARA GRUPOS VULNERÁVEIS : UM RELATO DE EXPERIÊNCIA DAS VIVÊNCIAS DE EXTENSÃO. Revista Brasileira de Tecnologias Sociais, v. 5, n.1 , p. 59-68, 2018.

46. BRASIL. Ministério da Saúde. Guia Alimentar para a População Brasileira. Brasília: Ministério da Saúde, n. 2, p. 1-158, 2014. 